\title{
SISTEMATIZACIÓN DEL PROCESO DE FORMACIÓN DE TRABAJADORAS SOCIALES EN EL MARCO DE LAS PRÁCTICAS INSTITUCIONALES: UNA APUESTA A LA CONSOLIDACIÓN DEL PROYECTO ÉTICO POLÍTICO PROFESIONAL.
}

\author{
SYSTEMATIZATION OF THE SOCIAL WORKERS TRAINING PROCESS
}

IN THE FRAMEWORK OF INSTITUTIONAL PRACTICES: A BET ON THE

CONSOLIDATION OF THE PROFESSIONAL POLITICAL ETHICAL PROJECT.

Por: Alba Lucía Marín Rengifo'

Jhon Sebastián Aguirre Cano ${ }^{2}$

Sandra Yaneth Vallejo González ${ }^{3}$

Recibido: 10 de mayo de 2019 - Aprobado: 14 de noviembre 2019

\section{RESUMEN}

La presente investigación ${ }^{4}$ obedece a una sistematización del proceso formativo de las trabajadoras sociales del Programa de Trabajo Social de la Universidad de Caldas, durante el desarrollo del componente curricular denominado, prácticas institucionales. Para ello realizamos una reconstrucción participativa de la experiencia vivida por las orientadoras de práctica, asesores institucionales, estudiantes y egresadas de dicho programa. Este artículo se estructura en tres componentes, inicialmente, hacemos referencia a las características generales que dinamizaron el proceso de sistematización como modalidad de investigación. Posteriormente, describimos los momentos que orientan el proceso de formación de la práctica institucional. Y finalmente, elaboramos una reflexión orientada a comprender las posibilidades de consolidación de un proyecto ético político profesional en la Universidad de Caldas, desde las concepciones de práctica institucional develadas: la práctica como aplicación rigurosa de la teoría; la práctica como construcción de la relación entre la identidad personal y profesional; y la práctica como proceso de reflexión en y sobre la acción.

Palabras clave: Prácticas institucionales, Trabajo Social, sistematización de prácticas,

\footnotetext{
${ }^{1}$ Trabajadora social, Magister en estudios de familia y desarrollo. Docente investigadora Universidad de Caldas. alba.marin@ ucaldas.edu.co

${ }^{2}$ Trabajador social, Magister en educación y desarrollo humano, estudiante Doctorado en Conocimiento y Cultura en América Latina. Docente investigador Universidad de Caldas. sebastian.aguirre@ucaldas.edu.co

${ }^{3}$ Trabajadora Social, Magister en ciencias sociales. Docente investigadora Universidad de Caldas. sandra.vallejo@ucaldas.edu.co ${ }^{4}$ Aclaramos que el artículo hace parte de la primera fase de la investigaciónn: Prácticas fundamentadas en el programa de trabajo social: una apuesta a la consolidación de proyectos ético-políticos profesionales orientada a documentar la experiencia de formación en práctica de trabajadores sociales de la Universidad de Caldas y su relación con la consolidación de proyectos ético-políticos profesionales.
} 
procesos de formación, proyecto ético-político profesional

\begin{abstract}
:
This research is a systematization of the teaching-learning process of the Social Work Program at the University of Caldas, concerning to the curricular element called academic practices. In order to do this, we carried out a participatory reconstruction of the experience lived by the practice counselors, institutional advisors, students and graduates of said program. This article has three parts, initially; we refer to the general characteristics that energized the systematization process understood as a research modality. Subsequently, we describe the moments that guide the formation process within the institutional practice. Finally, we elaborate a reflection aimed at understanding the possibilities of consolidating a professional political ethical project at the University of Caldas, from the conceptions of institutional practice revealed: practice as a rigorous application of the theory; practice as a relationship building between personal and professional identity; and practice as a process of reflection in and about action.
\end{abstract}

Key words: Institutional practices, social work, systematization of experiences, teaching-learning process, professional ethical-political project

\title{
1. INTRODUCCIÓN
}

Las prácticas institucionales en el Programa de Trabajo Social de la Universidad de Caldas, articulan las tres funciones misionales de esta institución: docencia, investigación y proyección. En este sentido, la intención de las prácticas no es solo la trasferencia o aplicación de conocimientos, sino la construcción y comprensión de las dinámicas inciertas de las realidades sociales, las experiencias de los actores involucrados en ellas y los contextos donde se configuran, entre otros aspectos. Gibbons, M., Limoges, C., Nowotny, H., Schwartzman, S., Scott, P., Trow, M., 1994, Acosta y Carreño, (2013).

Las prácticas institucionales como escenarios de construcción de conocimiento, nos implica considerar el proceso de formación de trabajadores sociales, en tanto comprendemos que la práctica obedece a un proceso de intervención, que supera la aplicación rigurosa de las teorías a partir de un método y se orienta por el reconocimiento de un campo complejo en el que se tensionan contextos, valores, fundamentos epistemológicos y teóricos, que hace inherente la investigación a la profesión. Matus, T., Onetto, L., Rodríguez, N., Paiva, D., Ponce de León, M., Quesada, M., (1995).

Las prácticas generalmente, se desarrollan en contextos institucionales en los cuales se construyen objetivos de transformación social y comprensión de los procesos sociales entre los diversos actores: estudiantes de práctica de los tres niveles (octavo, noveno y décimo semestre), profesoras orientadoras de práctica y sistematización y asesores institucionales; lo que exige de cada uno de ellos, una conversación permanente para construir sentidos colectivos frente a las intenciones que allí se persiguen. 
En concordancia con lo anterior y específicamente con la intención de aportar a la consolidación de un proyecto ético político profesional, dos años atrás un grupo de docentes del Programa de Trabajo Social de la Universidad de Caldas, emprendimos una sistematización de los procesos de enseñanza y aprendizaje en el componente curricular de prácticas institucionales. Entendemos los proyectos ético políticos profesionales, como la reflexión consciente que encarnan las comunidades académicas y organizativas alrededor de la representación de la profesión, los valores que la simbolizan; las finalidades que persiguen; los fundamentos teóricos y prácticos que la soportan; las normas y acuerdos para la acción; y el tipo relación que establece con las comunidades, instituciones (privadas y públicas) y otras profesiones. Netto, (2003).

Dado lo anterior, consideramos fundamental aportar al debate de la formación profesional en el Trabajo Social en Colombia, considerando su intención ético política. Lo ético, entendido como la reflexión a la cual está convocado cada sujeto profesional respecto a sus acciones en la sociedad, comprendiendo situaciones y contextos específicos, pero también los preceptos morales de la sociedad; y lo político, partir de los horizontes profesionales, que, en el caso del Trabajo Social, obedecen a la justicia social, la dignidad y la equidad.

En relación con lo anterior, asumimos la sistematización como una modalidad de investigación cualitativa, como una posibilidad de dar cuenta del desarrollo de los procesos formativos en el componente de práctica institucional de trabajadores sociales de la Universidad de Caldas, a partir del ordenamiento, recreación y cuestionamiento de los procesos construidos por los actores educativos, con el fin de obtener y develar conocimientos o aprendizajes, en torno al proceso de formación en la práctica, a partir de las experiencias y los sentidos atribuidos a este proceso. Guiso, (1998).

En el caso específico de esta sistematización, partimos por entender que las prácticas institucionales, están precedidas por lineamientos curriculares que se reconfiguran en las acciones singulares de los actores que interactúan en el proceso formativo, en este sentido, asumimos en esta investigación, que toda persona es sujeto de conocimiento en la medida que sitúe su reflexión, sobre la reflexión en la acción, en orientación no solo a las intenciones que persigue con la acción, sino en la forma como esta se da y lo que pasa posterior a ella. Larrosa, (2014).

En coherencia con lo anterior, afirmamos que el conocimiento trasciende la noción de información, puesto que implica, no solo la acumulación de datos frente a un fenómeno por parte de un sujeto, sino su comprensión en un contexto cambiante, a partir de sus procesos reflexivos, de análisis e interpretación, que a su vez influyen en su comportamiento, puesto que se asumen como propias las interpretaciones de dicha comprensión. En concordancia con Sousa (2008, p.6) el conocimiento "es una propiedad emergente/sistémica". 
Dado lo anterior, enfatizamos en el carácter participativo de la sistematización del proceso de formación de las prácticas institucionales, participación que no solo nutre el conocimiento construido por los sujetos, sino que nos exige establecer un diálogo permanente entre cada uno de los actores que configuramos el proceso de formación (orientadoras de práctica, orientadoras de sistematización, asesores institucionales, estudiantes y egresadas) para explorar los sentidos atribuidos al proceso de formación a partir de la comprensión de las situaciones particulares y de los roles cambiantes que se van asumiendo. Por tanto, el propósito de esta investigación, no fue traducir o interpretar las prácticas de los actores con relación al aprendizaje y a la enseñanza en el componente de práctica institucional, sino involucrarlos activamente desde sus construcciones y vivencias, en el proceso de reconocimiento de sus experiencias en el marco de la formación; en la reflexión sobre estas experiencias particulares; y en la generación de conocimientos orientados a los propósitos de la sistematización.

Nos encontramos en los procesos de sistematización con prácticas epistemológicamente vivas, que al disponer de todos los dispositivos para hacer que se constituyan en experiencias, el proceso de sistematización ayuda a construirlas, a hacer visibles sus finalidades, a mostrar la experiencia de los sujetos de la práctica, a construir el nudo relacional desde las acciones que la constituyen, que se forjan en la interacción del sujeto con el mundo, que hace que estos sean complejos, reflexivos, innovadores, empíricos, pero ante todo, cuando se colocan las posibilidades para realizarlo, son productores de saber y de teoría. Mejía, (2009, p.3)

Por otro lado, es necesario precisar que esta sistematización del proceso de formación de las prácticas institucionales de las trabajadoras sociales de la Universidad de Caldas, abarcó el período comprendido entre los años 2005 al 2013, lo que implicó ubicarnos en los currículos 316 y $447^{5}$ del Programa de Trabajo Social.

En el plan curricular 316 se planteaba que el objeto de Trabajo Social es el estudio y desarrollo de las interacciones y los procesos sociales en diferentes contextos y niveles de la realidad política, socio-económica, ambiental y cultural” (2001, pág.7). Este currículo cobraba vida a partir de siete núcleos ${ }^{6}$ de formación concebidos cómo ejes articuladores en la producción de conocimiento y proyección de la profesión. Cada núcleo se ubicaba en un espacio, un área, un campo o una unidad social de mediación de significados, a partir de un análisis de contexto en el que se fundamentaba el objeto particular, desde una perspectiva interdisciplinar, pero con orientación al Trabajo Social.

Por otra parte, en el plan curricular 447, se concibe que el objeto de Trabajo Social es "el conjunto de interacciones, procesos y estructuras sociales, en el marco del respeto a las diferentes formas de

\footnotetext{
${ }^{5}$ Actualmente vigente

${ }^{6}$ El currículo 316 planteaba siete núcleos en el componente de formación específica, así: 1 Trabajo social en el contexto de los procesos de construcción y constitución de lo humano y lo social. 2: Sociedad civil democracia y desarrollo sustentable. 3: Interacción de los procesos sociales y la problemática ambiental. 4: La salud integral como una condición básica para el desarrollo humano. 5 Familia, democracia, equidad y desarrollo. 6 : Trabajo y calidad de vida. 7: La proyección profesional: el diseño, la gestión, la evaluación y la sistematización de proyectos sociales
} 
vida y a los derechos humanos, hacia la construcción de democracia, convivencia y ciudadanía, en horizontes de justicia social, dignidad y equidad social”, Currículo del Programa de Trabajo Social, (2012, pág.15). Este currículo propone una formación fundamentada en el conocimiento y la reflexión crítica de la realidad, a partir de aspectos teórico prácticos de la intervención profesional con sujetos, familias, grupos, comunidades y organizaciones sociales, como ámbitos de actuación profesional.

Los participantes en la sistematización de las prácticas institucionales fueron los orientadores de práctica, los asesores institucionales, las estudiantes y egresadas ${ }^{7}$ que hicieron parte de la formación durante el periodo de tiempo seleccionado. Se configuró un grupo de participantes con quienes se realizó el proceso de reconstrucción, reflexión y comprensión de la experiencia vivida durante el desarrollo del proceso de formación en las prácticas institucionales.

Al inicio de esta sistematización, se realizaron jornadas de presentación de la propuesta, para vincular a los participantes e incluir aspectos considerados por estos necesarios y de interés colectivo. Después se realizaron entrevistas semiestructuradas individuales y conversatorios grupales, que fueron demarcando la reconstrucción del proceso de formación desde lo experimentado por los diversos actores. Posteriormente, se socializó la reconstrucción del proceso por medio de una línea del tiempo, que nos permitió hacer un análisis participativo entre los diferentes grupos de actores (docentes, asesores institucionales, egresadas y estudiantes) que demarcó la construcción final de una propuesta de diplomado con la que pretendimos ampliar nuestros conocimientos y comprensiones respecto a las prácticas formativas de trabajadores sociales y a la articulación entre el desarrollo de prácticas institucionales y el ejercicio profesional. Para efectos de este artículo, nos detendremos en un primer momento de la sistematización, el cual obedece al ordenamiento, reconstrucción, reflexión y comprensión de los procesos de formación de trabajadores sociales en el Programa de Trabajo Social de la Universidad de Caldas, durante el desarrollo del componente curricular, denominado prácticas institucionales.

\section{Reconstrucción de la práctica institucional}

El proceso de desarrollo de la práctica institucional, da cuenta de seis momentos identificados como significativos por profesoras orientadoras de práctica, asesores institucionales, egresadas y estudiantes ${ }^{8}$ de octavo, noveno y décimo semestre del Programa de Trabajo Social de la Universidad de Caldas. Los momentos hacen referencia a lo vivido previó a la práctica institucional, durante la misma y después de ella. A continuación, describiremos cada uno de estos momentos.

\footnotetext{
${ }^{7}$ La conformación del grupo de participantes tuvo en cuenta instituciones y egresados del Programa de Trabajo Social de la Universidad de Caldas, ubicados en el departamento de Caldas.

${ }^{8}$ En los currículos 316 y 447 del Programa de Trabajo Social de la Universidad de Caldas, se establecen tres niveles de práctica, así: I nivel, octavo semestre; II nivel, noveno semestre; III nivel, décimo semestre.
} 


\subsection{El proceso formativo que antecede el desarrollo de la práctica institucional}

El proceso previo al desarrollo de la actividad académica práctica institucional $\mathrm{I}^{9}$, es reconocido por profesoras, estudiantes y egresadas, como el momento formativo en el que se fundamenta teórica y metodológicamente la práctica. El proceso formativo se soporta en la estructura de los currículos de Trabajo Social, a los que hace referencia esta sistematización, el currículo 316 y el 447. En ambos currículos, reconocemos la desarticulación existente entre los fundamentos de las ciencias sociales y las áreas de intervención del Trabajo Social o las metodologías de intervención.

En el currículo 316, el desarrollo de los núcleos se dinamizaba mediante una estrategia teórico-práctica que permitía a las estudiantes involucrarse paulatinamente en el contexto y vivir experiencias de mediación con el mismo, a través de las preprácticas, en las cuales se construía un microproyecto. Posterior al desarrollo de los núcleos, las estudiantes cursaban la profundización de su interés: familia y desarrollo humano, salud, laboral, medio ambiente, organizaciones sociales, o la profundización de niños, niñas y jóvenes desvinculados del conflicto $\operatorname{armado}^{10}$, de acuerdo a los núcleos vistos, profundizaciones que generalmente definían la asignación de práctica.

En el año 2011 se construyó el currículo 447 en el programa de Trabajo Social, con énfasis en las áreas de investigación y de metodologías de la intervención. Frente a los procesos de preprácticas desarrollados en las actividades del componente de metodologías de la intervención, se pretende que las estudiantes apliquen los conocimientos teóricos de los métodos de intervención a través del desarrollo de microproyectos.

Reconocer la fundamentación y estructura de los currículos 316 y 447 en este proceso de sistematización, permitió identificar dos características curriculares que median la formación antes del desarrollo del componente de práctica institucional, la primera, vinculada con la separación de la teoría y la práctica; de la intervención y la reflexión; y la segunda, relacionada con el orden jerárquico entre teoría y práctica, estructurando una relación secuencial, en dónde la teoría antecede necesariamente a la práctica en los procesos formativos.

La fundamentación de la práctica se entiende como un momento de preparación para enfrentar y conocer la realidad, una realidad que parece lejana a los objetos de estudio de las disciplinas y específicamente al objeto de Trabajo Social. La teoría cobra sentido cuando esta tributa a la orientación, comprensión y construcción de la realidad social y a las formas de hacer Trabajo Social.

${ }^{9}$ El plan de estudios del Programa de Trabajo Social en la Universidad de Caldas consta de diez semestres, en que se identifican cuatro componentes fundamentales que guían la formación: formación general: como opción para el fortalecimiento ético, estético o ciudadano del estudiante; formación en ciencias artes o filosofías: desde una perspectiva interdisciplinar comprende fundamentos de diferentes disciplinas de las ciencias sociales como apuesta de un trabajador social que cuestiona la realidad y propone alternativas de acción y política pública que apunten a los derechos humanos y las diversas formas de vida; formación especifica: en que encontramos tres áreas específicas, fundamentación (núcleos para el currículo 316 y metodologías de la intervención, planeación, administración, y gestión del desarrollo social para el 446), investigación social y sistematización de la práctica, además de prácticas en tres niveles. Y profundización: como la posibilidad de las estudiantes para adquirir mayores conocimientos teóricos y metodológicos en torno al trabajo social y a la profesión.

${ }^{10}$ La profundización de niñas, niños y jóvenes desvinculados del conflicto armado se consolidó en el proceso de transición del currículo 316 al 447. 
De esta manera, la teoría se apropia cuando existe la posibilidad de ampliar comprensiones frente a fenómenos o situaciones cotidianas y cercanas. Por su parte, los asesores institucionales desconocen el currículo del programa de Trabajo Social y su objeto, parte fundante de la formación en la práctica, sin embargo, reconocen que las estudiantes ingresan a este proceso, con una serie de conocimientos que aportan desde lo teórico y metodológico a los procesos institucionales.

Se espera según coinciden profesoras y asesores institucionales que las estudiantes al iniciar la práctica, sean las encargadas de hacer las articulaciones, entre los conocimientos adquiridos en la formación universitaria y las realidades en las cuales van a desarrollar sus procesos de intervención.

\subsection{La selección de las áreas o campos de intervención profesional}

La selección de las áreas o campos de intervención para la ubicación de las estudiantes en práctica, es un momento que se vive con expectativa, generando ansiedad y nerviosismo en las practicantes, en tanto implica tomar decisiones respecto a la población con la que se va a trabajar, las posibilidades de construir opciones laborales, y de avizorar condiciones básicas que garanticen el desarrollo del proceso.

La Universidad de Caldas, cumple con garantizar el campo de práctica donde las estudiantes desplieguen sus conocimientos a partir del diseño, ejecución, evaluación y sistematización de un proyecto social, el cual se convierte en la brújula que orienta la relación formativa entre estudiantes y orientadoras. La selección del campo de práctica es un momento trascendental que nos invita a construir intereses colectivos entre los diversos actores, en tanto se constituye en la posibilidad de gestar apuestas éticas y políticas fundadas en el objeto del Trabajo Social, a partir de esta reflexión, surgen las siguientes preguntas: ¿Cómo construir procesos colectivos de selección de los campos de práctica en los que se tengan en cuenta intereses profesionales, personales, institucionales y de las comunidades que se oriente hacia la justicia social? y ¿Qué criterios deberíamos tener en cuenta en los procesos de articulación de dichos intereses?

\subsection{La ubicación de las estudiantes en el campo de intervención profesional}

La ubicación institucional, se asume como el paso del mundo académico al acercamiento, interacción y comienzo de construcción de relaciones de estudiantes, orientadoras de práctica y sistematización, asesores institucionales, poblaciones y otros profesionales. Para las estudiantes se traduce en el contacto con la realidad y el despliegue de las capacidades teóricas y metodológicas a través de un proyecto social.

Las prácticas institucionales entre universidad y la institución, están mediadas por convenios interinstitucionales, los cuales orientan la definición y el desarrollo de intencionalidades, actividades, funciones, perfiles y compromisos, de acuerdo al rol que tiene la institución, la Universidad y las 
Alba Lucía Marín Rengifo - Jhon Sebastián Aguirre Cano - Sandra Yaneth Vallejo González

practicantes; lo que señala el inicio de una relación formal entre asesor, docente y estudiante, caracterizada por tres acuerdos básicos: el primero relacionado con el desarrollo de un proyecto social que responda a los intereses de la institución y a la perspectiva del área de intervención profesional, para ello, la institución proporciona la ubicación en un contexto determinado, indicando la población, los recursos y el acompañamiento del asesor institucional. El segundo, hace referencia al compromiso de la estudiante de realizar un apoyo institucional, en programas, proyectos y acciones propias que contribuyan a dinamizar, descongestionar y/o dar cumplimiento a las demandas que exigen contratos y alianzas que tienen las entidades. El tercer acuerdo, se orienta a la garantía de la ejecución de un proyecto social, que se constituye en la posibilidad de potenciar competencias profesionales en las estudiantes, tales como, el trabajo con las comunidades, la planeación y gestión de procesos que contribuyan al acercamiento, el conocimiento y desarrollo de propuestas de intervención, que posteriormente serán evaluadas desde criterios académicos.

Las estudiantes reconocen que ingresar a la práctica y ubicarse en un campo de actuación profesional, implica necesariamente "confrontarse con la realidad" proceso de aplicación de conocimientos, sino de generación de este, sustentado en las dinámicas particulares del contexto y en el encuentro y reconocimiento de la voz de los actores sociales $e$ institucionales con los que interactúa.

En síntesis, la relación inicial de conocimiento, acercamiento y acuerdos entre la Universidad y los campos de práctica en el momento de la ubicación institucional, nos señalan vacíos, tensiones, encuentros y desencuentros, que nos permiten interrogar el proceso formativo respecto a ¿Qué lugar tiene el estudiante en la institución? ¿Cuál es el rol formativo de las instituciones en los procesos de práctica? ¿Qué sabe la institución de la intencionalidad del Trabajo Social desde el objeto profesional planteado por el Programa? y ¿Qué se transforma con el desarrollo de los proyectos sociales, en las dinámicas institucionales?

\subsection{El diseño del proyecto social}

Los proyectos sociales los entendemos como una herramienta de planificación o una alternativa de intervención a partir del cual se articulan intereses y se dinamizan las relaciones entre las instituciones y la Universidad, con el propósito de aportar a las condiciones de vida de los sujetos, grupos, comunidades y organizaciones, en orientación a la justicia social, dignidad y equidad. El diseño del proyecto, pone en cuestión el principio de flexibilidad y articulación entre teoría y práctica; la apropiación del objeto de Trabajo Social; la concepción de la planeación; el lugar de los conocimientos, las experiencias y las necesidades sociales; y las apuestas profesionales que se vienen gestando en el proceso de formación. En esta sistematización, el proyecto lo hemos asumido

\footnotetext{
${ }^{11}$ Expresión comúnmente utilizada por las estudiantes cuando ingresan a práctica.
} 
desde tres lógicas configuradas a partir de las áreas de formación o de los colectivos de trabajo, así:

\subsubsection{El proyecto como foco de la orientación de la práctica institucional}

La orientación se focaliza, en el interés porque las estudiantes, reconozcan y experimenten el ciclo de un proyecto social, en tanto, su diseño, ejecución y evaluación. De esta manera, en nivel I de práctica las estudiantes diseñan un proyecto social, para lo cual es indispensable realizar un proceso diagnóstico, en el que se indaga la realidad, estableciendo una relación directa con la población a partir de la conversación y el cuestionamiento de situaciones concretas. El diagnóstico fundamenta la práctica, visibilizando la relación investigación e intervención. En el nivel II y III ejecutamos y evaluamos el proyecto, para lo cual es indispensable que las estudiantes puedan dar cumplimiento al desarrollo de las actividades programadas en el proceso metodológico propuesto en el I nivel de práctica. En esta lógica, resaltamos el papel de la vigilancia epistemológica en la construcción del proyecto, considerada por algunas profesoras, como la posibilidad de garantizar la coherencia de la propuesta, en cuanto a la problemática identificada, paradigma, modelo, alternativa y estrategia de intervención.

Entender el diseño del proyecto como el foco de la orientación de la práctica, nos ha llevado a caer en ocasiones en lógicas de intervención instrumental, en donde las estudiantes logran desarrollar el proyecto social, pero con dificultades para comprender y aprehender los procesos sociales a partir de su reconstrucción y cuestionamiento de la práctica formativa y de los aprendizajes allí obtenidos.

\subsubsection{La práctica como interés académico y apoyo institucional}

La institución reconoce su participación en la gestión de recursos para el desarrollo del proyecto, en actividades puntuales en el proceso de diagnóstico y en la presentación del proyecto social construido por las estudiantes, en relación con las necesidades o características particulares de la población. La participación que tiene la institución en el diseño del proyecto social, es generalmente de espectadora, financiadora y/o evaluadora del proyecto.

En el apoyo institucional se regula las actuaciones de las estudiantes con relación a los intereses, demandas y dinámicas propias de la institución. Este apoyo se orienta a: realizar el registro de actividades, acompañar procesos de caracterización de poblaciones, favorecer procesos de ordenamiento de información y cubrir actividades en ausencia del titular, entre otros. Aspectos reconocidos por sus aportes a la formación, en tanto contribuyen a situar el lugar del Trabajo Social en la institución, sus roles y afianzar la identidad profesional, esta situación entra en tensión cuando además del apoyo institucional, las estudiantes deben responder a los compromisos académicos asignados. 


\subsubsection{El proyecto como orientador del proceso formativo en la práctica}

Se entiende que el proyecto social es una responsabilidad de las estudiantes; ellas son las encargadas de garantizar la construcción y el desarrollo de una propuesta de intervención. La responsabilidad de la orientadora radica en acompañar este proceso a partir del cuestionamiento y reflexión constante de las situaciones que emergen en este recorrido, de tal manera, que las estudiantes se formen y se reconozcan profesionalmente a través de las dinámicas particulares que les demanda el desarrollo del ciclo del proyecto. Se trata de construir una apuesta profesional que impacte tanto el proceso formativo, como el proceso social y la dinámica institucional.

\subsection{La sistematización de la práctica institucional ${ }^{12}$}

Este proceso es comprendido por los orientadores de las actividades académicas sistematización I y sistematización II como la interpelación a la práctica institucional y profesional; la emergencia de la pregunta como movilizadora del pensamiento en tanto contribuye a reconstruir, comprender y crear alternativas de intervención profesional; así como también, una posibilidad para formar un profesional reflexivo.

En cuanto a las instituciones, no se evidencia un reconocimiento del proceso de sistematización, se considera que este es un requerimiento definido por la academia, por el cual las estudiantes asumen nuevas responsabilidades de mayor tensión, ocupación de tiempo y énfasis escritural. Sin embargo, cuando las instituciones reconocen las intenciones de este proceso formativo a través de las mismas estudiantes o de las orientadoras de práctica, generalmente expresan su interés por ser parte de este.

Este momento es reconocido generalmente por las estudiantes por su posibilidad de interpelar la práctica; de construir una experiencia formativa que genera aprendizajes, personales, profesionales y sociales. La sistematización es entendida como la oportunidad de cuestionar la intervención profesional, se trata de una exigencia a pensar la profesión y a asumir una posición crítica sobre la realidad social y el papel del Trabajador Social en la misma. Sin embargo, para algunas estudiantes, la sistematización es una actividad académica que genera temor, angustia, frustración, siendo en ocasiones denominada por las mismas estudiantes como "traumatización", asociando a esta denominación, las dificultades para desarrollar paralelamente el proceso de práctica y sistematización, en cuanto al tiempo y a la comprensión de los procesos sociales, por parte de las orientadoras de práctica y profesoras de sistematización. En este sentido surge la pregunta ¿Quiénes deben comprender el proceso social?

\subsection{La evaluación de la práctica}

Se referencia la evaluación de la práctica como un momento significativo, en tanto este tiene que

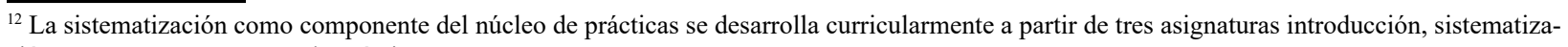
ción I y II, estas acompañan la práctica I, II y III. 
ver con la definición de si se aprueba o no esta actividad académica, pero también, con el reconocimiento de los logros, avances y aprendizajes construidos en este proceso. La evaluación es generalmente realizada a partir de las dimensiones del ser, el conocer y el hacer de las estudiantes, respecto al diseño y desarrollo de proyecto social. Para las estudiantes el objetivo de la evaluación es ganar la práctica y reconocer los logros y limitaciones del Trabajador Social en formación.

El proceso de evaluación está regido generalmente por instrumentos con criterios cuantitativos y cualitativos. La mayoría de orientadoras plantean duda al respecto, consideran que las dificultades de la evaluación están asociadas al énfasis que hacemos las orientadoras en los instrumentos, perdiendo de vista las situaciones concretas que se dan en la relación formativa.

Por su parte los asesores institucionales y estudiantes, consideran que en el proceso evaluativo la institución deberían tener mayor participación. Sin embargo, reconocemos por parte de las orientadoras de práctica que los criterios de las instituciones distan de los criterios de evaluación orientados en la Universidad.

Comprendiendo el hilo descriptivo que se viene planteando surgen preguntas como: ¿Cuál es el sentido que soporta la aproximación de las practicantes a las comunidades? ¿Qué intenciones se persiguen en estas relaciones no solo con las comunidades sino con otras disciplinas? y ¿Cuáles son los criterios que deben considerarse en la evaluación de un proceso de práctica?

\section{Aprendizajes y hallazgos}

A continuación expondremos los aprendizajes y hallazgos frente a los procesos de formación en el marco del desarrollo de las prácticas institucionales en el Programa de Trabajo Social. Para ello, plantearemos la reflexión respecto a las concepciones de la orientación de la práctica institucional y su relación con las posibilidades de consolidación de un proyecto profesional en la Universidad de Caldas.

\subsection{Concepciones de la orientación de la práctica institucional y aproximaciones para la con- solidación de un proyecto ético político profesional.}

Comprendemos que la construcción de un proyecto ético político profesional es una tarea ineludible para el Trabajo Social en Colombia, ya que es en esta construcción donde se recogen las discusiones en torno a los valores que nos representan en la sociedad; los fines y funciones que tenemos como profesionales; los aspectos teóricos y prácticos que sustentan la praxis profesional; y las normas o formas de relacionamiento desde las cuales vamos a interactuar en diferentes contextos, de allí la relevancia, de reflexionar sobre los procesos de formación profesional de estudiantes de Trabajo Social.

El carácter personalizado de la formación en la práctica y sus complejas exigencias, nos ha permitido identificar dos formas de asumir este proceso pedagógico: por una parte, entendido 
como acompañamiento, y por otra como orientación; estas formas no se presentan estáticamente, por el contrario, permanentemente mutan y se imbrican en la relación con las estudiantes.

El acompañamiento en el proceso formativo, enfatiza en el interés porque el estudiante construya una relación entre su subjetividad y el Trabajo Social. Desde esta concepción se sitúa al estudiante en el centro de la formación y ubica a la profesión como actor de reparto en este proceso. En consecuencia, la complejidad del objeto profesional y sus horizontes de justicia social, dignidad y equidad, tensan la relación entre intervención e investigación. Desde esta concepción la formación es asumida como el desarrollo de competencias en el ser, hacer y conocer, dando relevancia a la dimensión ser.

En lo correspondiente a las formas de evaluar, el acompañante, parte de un marco cualitativo y subjetivo, donde no se consideran criterios o herramientas construidas previamente para llevar a cabo la evaluación, ya que esta depende de la singularidad de la estudiante y de la relación que construya con la misma. En este proceso suele darse una contradicción entre el exitoso cumplimiento de las labores en el campo de práctica y un escaso rendimiento académico.

La orientación en el proceso formativo, demarca otros intereses en la formación de las estudiantes, además de su identidad personal. Por una parte, la apropiación temática y teórica, en el abordaje del objeto profesional y por otra, la importancia que se le asigna a la capacidad reflexiva de las estudiantes para articular los procesos de investigación y de intervención comprendiendo las interacciones que configuran la acción profesional. Desde esta orientación, las docentes, le asignan mayor trascendencia al saber profesional, situándolo a la par del saber pedagógico; es decir, el docente recupera la dimensión pedagógica de la enseñanza en la formación, lo que le implica la pregunta por la enseñanza del conocimiento disciplinar o profesional.

En coherencia con lo anterior, esta sistematización nos permitió evidenciar una relación directa entre las experiencias de formación en y para la práctica de cada uno de los actores que participaron en este proceso y los aportes a la consolidación de un proyecto ético político profesional en la Universidad de Caldas, el cual se configura en el reconocimiento de la diversidad de actores, intereses y propósitos construidos previa y simultáneamente al desarrollo de las prácticas institucionales. En consecuencia, evidenciamos tres concepciones de la práctica institucional que dinamizan el proceso formativo:

La primera concepción, asume la práctica institucional como la aplicación rigurosa de la teoría, en la que se focaliza la orientación en el saber experto de profesoras y estudiantes en un campo temático específico. Esta orientación, se dinamiza a partir del reconocimiento de los insumos previos al componente de práctica y de la orientación del proyecto social como foco central de la formación académica. 
Desde esta concepción se construye una relación entre las instituciones y el programa, marcada por la división de funciones que fragmenta la realidad, en donde las estudiantes son especialistas en áreas temáticas y los asesores institucionales en el conocimiento de la realidad, esta situación demarca roles y ámbitos específicos de acción, que si bien, le posibilitan al estudiante una identificación mucho más concreta de lo que debe hacer, no contribuyen en la articulación , y con ello una ubicación contextualizada de la realidad social. Desde esta perspectiva, el orientador requiere un conocimiento amplio frente a la temática, más que en lo referente al saber profesional, este conocimiento debe estar construido principalmente desde su experticia y actualización permanente de tal manera que le permitan avizorar cuáles son las demandas del medio y las diferentes vertientes teórico metodológicos desde las cuales intervenir en un tema específico.

Por otra parte, desde esta concepción se exige la ubicación en un paradigma específico que le permita a la estudiante modelar la realidad a abordar, disponiendo de una serie de herramientas para construir el proyecto social y con ello mantener correctamente su andadura por el paradigma, de manera que la realidad se adapte a la visión paradigmática. A partir de las herramientas el docente instaura una vigilancia epistémica permanente para que el estudiante no pierda de vista el paradigma. En concordancia con lo anterior una orientadora de práctica refiere:

La formación en visiones paradigmáticas se hace fundamental, porque implica, cada que hacemos ese proceso, de inducción a la práctica, se requiere ese fundamento de la perspectiva nuestra que trabajamos para poder en un ejercicio de acompañamiento, poder orientar al estudiante de entrada en esa visión paradigmática de como él va a querer ver esa realidad sobre la cual se va articular, eso se hace fundamental; la formación uno diría que también el manejo de los instrumental, el manejo de lo metodológico, que uno sabe que por sí solo son neutros, pero entonces usted con esa perspectiva paradigmática usted le da a eso sentido, le da una finalidad.

PGLA, (2015, p 10)

Como se puede evidenciar, el paradigma es el que le da fundamento al estudiante para construir la mirada de su práctica y por tanto de la realidad que pretende abordar, esta se configura a priori de su contacto con ella, porque necesita de unos lentes que le permitan enfocarla en las cuestiones que le deben interesar de acuerdo a lo que esa forma de modelar la realidad exige.

Si bien es innegable el aporte de esta concepción de formación al estudiante, dado el camino expedito que le ofrece, pueden perderse situaciones de la realidad que no están contenidas en el paradigma y por la escasa fortaleza que trae el estudiante en leer reflexivamente la teoría, termina moldeando o encajando las realidades a esta, cuando esto no puede darse surge una situación de tristeza, de impotencia que no es abordado abiertamente por el estudiante, sino que entra en un clima de desesperanza que ubica al objeto profesional como un ideal que difícilmente puede ser cumplido. 
En este sentido, puede entenderse que la práctica es producto de una reflexión ética y política, en tanto está determinada por la razón, que apela a la ciencia como estatuto para validar el conocimiento desde el cual se orienta, poniendo de lado a los sujetos que hacen parte del proceso y a los contextos dónde se produce el conocimiento, apelando a un criterio de universalidad. Bajo esta idea, las concepciones de práctica construidas por docentes y estudiantes en la formación, se quedan en una perspectiva prescriptivista de la ética puesto que las acciones son evaluadas por las reglas o normas que la guían y por tanto en su correcta aplicación ${ }^{13}$. Hare, (1999)

Caben en esta concepción, aspectos como la consistencia lógica de la intervención que implica demarcar un paradigma que define la problemática identificada, el modelo, la alternativa y la estrategia que guían la intervención, de esta manera, la construcción de la realidad queda limitada por un a priori moral que no interpela la ciencia como discurso de saber, ni el papel que ha tenido el Trabajo Social en la ciencia y en su configuración como profesión.

La segunda concepción de la práctica, está orientada por la construcción de la relación entre la identidad personal y profesional de las estudiantes. Desde esta concepción, se resalta el papel fundamental que juega la identidad profesional construida previamente al proceso de práctica, como el interés por consolidarla a partir del acercamiento a la identidad personal de las estudiantes. La tensión en esta concepción, surge cuando el proceso de orientación se centra en la identidad personal de las estudiantes y se pierde de vista, el proceso de formación en la práctica, en donde confluyen los tres ejes misionales de la Universidad: los intereses de la población y de la institución que hace parte del proceso; y los principios teleológicos de la profesión, orientados a la justicia, la dignidad y la equidad. En las conversaciones que realizamos como orientadoras de práctica, una docente expresó:

Porque yo creo que la formación tiene que ver mucho con la identidad, con quienes sí sentimos y vivimos el Trabajo Social, esos son los que llegan con mayor apropiación y claridad. Pero quienes aún llegan como buscándole sentido a la profesión, esos son los que de pronto uno ve como más desmotivados, y por tanto esos no la tienen tan clara, porque no hay un interés frente a eso. PDI, (2015. pág. 5)

Desde esta concepción de la orientación de la práctica, se rompe con las bases prescriptivistas de la ética basada en la ciencia, sin embargo, se enfrenta a la dificultad de asumir una perspectiva subjetivista, en dónde la relación con la práctica está determinada por las necesidades y condiciones de

\footnotetext{
${ }^{13}$ Cortina y Martínez (1998) a propósito de la ética prescriptivista expresan "La primera característica del lenguaje valorativo es que prescribe la conducta, la orienta aconsejando o mandando, pero siempre apoyándose en razones, que, en principio, se originan en las características del asunto que se juzga y que pueden ser expresadas mediante lenguajes descriptivos. Para explicar por qué algo es bueno es preciso describir las características que lo hacen apreciable y que sintonizan con el paradigma que de ello se tiene, de modo que se presente como recomendable" (Ética Akal editorial. Madrid España).
} 
vida particulares de los estudiantes y docentes y no, de una perspectiva colectiva de la profesión. Adela Cortina se refiere a esta ética como neoconservadora y la describe de la siguiente forma:

los rasgos del nuevo modo de pensar y sentir, por contraposición con el orden sistemático moderno, serán el fragmentarismo, la negativa a armonizar lo universal y lo singular, optando por lo singular, la superación de la idea de fundamento, la aceptación del pluralismo, la indeterminación y las diferencias, el anuncio del fin de los grandes relatos como legitimadores del saber científico, la aceptación del caos -no del cosmos y del progreso-, la sustitución de la epistemología por la hermenéutica, la firma del certificado de defunción de la filosofía de la historia. Cortina, (1990, p125).

Bajo esta concepción, la reflexión ética de la formación pasa de un extremo cientificista a otro en el que no hay un fundamento para la práctica, sino emociones que se deben atender, dando relevancia a la dimensión del ser, la cual, a su vez, se constituye en uno de los principales intereses que median la relación con los campos de práctica.

Frente a la relación entre la práctica formativa y la identidad profesional, Cifuentes expresa:

Tales características no liberan a los programas académicos de Trabajo Social de la responsabilidad que les corresponde en la consolidación de las identidades profesionales; por el contrario, los compromete con el desarrollo de proceso en los cuales las estudiantes y las estudiantes entren en contacto reflexivo con la profesión y despliegan sus potencialidades de análisis, investigación, crítica, construcción, organización, producción y proyección social. Experiencias formativas que involucren sus voces en diálogos con sujetos y colectivos en los cuales se proyecta, se hace su debate y se reconstruye la profesión (2011, pág.196).

La tercera concepción, asume la práctica institucional como una apuesta formativa de trabajadores sociales, preparados para afrontar y desempeñarse profesionalmente en contextos complejos, dinámicos, cambiantes y contradictorios que demandan la construcción de procesos creativos, innovadores y pertinentes a la realidad social. Desde esta lógica, se supera la condición personal del estudiante y del conocimiento profesional, centrado en discursos y herramientas y se vuelve fundamental el conocimiento colectivo y práctico que emerge de los procesos de interacción, entre los actores sociales, institucionales y académicos. En esta concepción, se asume que el uso racionalizador, acrítico, descontextualizado y dogmático que el Trabajo Social ha hecho de las teorías sociales, asignando un activismo y pragmatismo a la profesión que se traduce en falta de autonomía frente a las imposiciones institucionales, predominio de la perplejidad para enfrentar la incertidumbre y el desplazamiento del conocimiento como orientador y guía.

La concepción de la práctica institucional como escenario de formación que supera una mirada racional, técnica e instrumental de la misma, cuestiona asuntos que tienen que ver con la manera 
Alba Lucía Marín Rengifo - Jhon Sebastián Aguirre Cano - Sandra Yaneth Vallejo González

como asumimos el Trabajo Social y con ello la formación de trabajadores sociales comprometidos con la transformación de la realidad social, en la perspectiva de justicia social, dignidad y equidad social. En este sentido, Cifuentes y Vallejo (2018, pág.155) plantean

Cuando como trabajadoras sociales que participamos de la formación de las nuevas generaciones de profesionales nos enfrentamos a la reflexión acerca de los retos que el momento histórico nos está planteando, nos vemos compelidas a buscar en la historia del Trabajo Social claves para esclarecer nuestra responsabilidad social, en tanto profesión que se interpela desde la justicia como principio, como fin, como enfoque, como campo de investigación y de acción profesional.

Este reconocimiento, nos permite a la vez comprender cómo leemos y comprendemos los contextos sociales y las formas particulares en las que nos permitimos reconstruir y aprender de la práctica profesional. Esta concepción de la práctica, nos invita a repensar en la formación de un profesional reflexivo, como aquel profesional que, según Mendoza, citado por Cifuentes (2005, pág.19), "interpreta su realidad, tiende a realizar un diálogo interpretativo con las situaciones que interviene, es crítico, crea y experimenta constantemente distintas estrategias de acción, y reformula las explicaciones que hace de la realidad"

Las concepciones que las profesoras hemos construido frente a la orientación de la práctica institucional, tiene que ver con la manera como asumimos, nuestra práctica docente, la cual generalmente está caracterizada por la complejidad, particularidad e incertidumbre; condiciones que no son fáciles de comprender en un proceso pedagógico caracterizado como lo plantea Ranciere por un "mundo dividido en espíritus sabios y espíritus ignorantes, espíritus maduros e inmaduros, capaces e incapaces, inteligentes y estúpidos" (2003, pág.8).

En este sentido, el ejercicio docente nos cuestiona sobre nuestro lugar y el lugar de los otros en los procesos formativos, en tanto inciden directamente en los procesos de formación. Schön (1992, pág.10) plantea que en los procesos formativos existe de manera permanente el dilema del rigor o la pertinencia, lo cual tiene que ver con la epistemología de la práctica en la que se pone en relación la competencia en la práctica y el conocimiento profesional. De igual manera expresa

No deberíamos empezar por preguntar cómo hacer un mejor uso del conocimiento científico, sino qué podemos aprender a partir de un detenido examen de arte, es decir, de la comprensión por la que en realidad los prácticos somos capaces de manejar las zonas indeterminadas de las prácticas independientemente de aquella otra competencia que se puede relacionar con la racionalidad técnica

Desde esta concepción de la práctica institucional, se propone que la formación profesional más que conducir a que el estudiante se especialice en un tema o entienda teóricamente el objeto profesional, pueda construir un proceso en el que a partir de su práctica institucional, articule la 
formación, la proyección y la investigación. Para ello se vuelve fundamental que el docente potencie las capacidades reflexivas del estudiante en lo que plantea como profesional reflexivo.

La práctica se configura en una zona indeterminada, que involucra formarse, a partir del ejercicio profesional y al mismo tiempo construir conocimiento de manera que la práctica pueda realimentarse no solo para el aprendizaje del estudiante, sino para encontrar mejores alternativas a las cuestiones que propone la realidad o de construcción de las realidades.

Estas zonas indeterminadas implican para las docentes asumir un lugar de incertidumbre en el que cada estudiante y proceso de práctica plantean retos distintos, pero que al mismo tiempo, lo ubican en una finalidad respecto a la formación profesional, orientada por la reflexividad de las interacciones que como trabajadores sociales se construyen en el contexto particular de intervención y la provocación por construir conocimientos derivados de la acción. En este sentido, una orientadora de práctica plantea:

...yo quisiera con los estudiante en práctica, que aprendan, de todo lo que hay que aprender que es mucho; que se encuentren de frente con su profesión, que se piensen como trabajadores sociales, que se hagan cargo de eso que está pensando, y eso que piensan implica otro punto, que se hagan responsables de las implicaciones de actuar profesionalmente, que es la implicación de investigar y de producir conocimiento, de aportarle a una comunidad académica, pero que es una responsabilidad social en la que está inserta su labor, hay que tomárselas en serio, para mí eso es muy importante, que reflexione cada cosa que hacen, que discutan, que se enriquezcan, pero en últimas es que se haga responsable de la realidad social en la que están ... PRO, (2015.pág. 7)

Desde esta orientación la práctica de las estudiantes se configura a partir de tres líneas fundamentales, la acción profesional, la reflexión permanente de ella y la construcción de conocimiento a partir de la reflexión en la acción. La vinculación de estas tres líneas en la práctica, demanda una estudiante que se pregunte constantemente y que tenga la capacidad de hacer una mirada integradora entre lo que hace y lo que piensa en horizonte de los principios teleológicos profesionales, lo que supone la construcción de apuestas profesionales y ciudadanas que promuevan la participación social como posibilidades de encuentro, de diálogo y de movilización social en orientación a las realidades sociales que configuran el contexto colombiano.

La acción profesional, la reflexión y la construcción de conocimiento sobre los principios orientadores del Trabajo Social, se constituyen en asuntos claves en el proceso de orientación de las prácticas institucionales, en tanto se orientan a la formación de ciudadanos críticos y activos, que a su vez contribuyen a aportar desde procesos participativos de intervención profesional, a la politización de la vida pública. 
Alba Lucía Marín Rengifo - Jhon Sebastián Aguirre Cano - Sandra Yaneth Vallejo González

\section{REFERENCIAS BIBLIOGRÁFICAS}

Acosta, W y Carreño, C. (2013). Modo 3 de producción de conocimiento. Implicaciones para la Universidad de hoy. Revista de la Universidad de la Salle, 61, 67-87. https://revistas.lasalle.edu. co/index.php/ls/article/view/243

Cifuentes, R. (2005). "Para provocar la conversación: La práctica en la formación de los trabajadores. Documento de trabajo". Manizales Universidad de Caldas.

Cifuentes, M. (Ed). (2011). Identidad y formación en Trabajo Social. Memoria I. Seminario Internacional, Buenos Aires: Lumen.

Cifuentes, M. R. y Vallejo, S. Y. (2018). Trabajo Social y justicia social en tiempos de transición. Revista Eleuthera, 18, 150-165. DOI: 10.17151/eleu.2018.18.9.

Cortina, A. (1990). Ética sin moral. Madrid, España: Tecnos.

Cortina, A. y Martínez, E. (1998). Ética. Madrid, España Akal Editorial.

Currículo de Trabajo Social 316 (1998) Documento de circulación interna. Universidad de Caldas.

Currículo de Trabajo Social 447 (2012). Documento de circulación interna. Universidad de Caldas.

Gibbons, M., Limoges, C., Nowotny, H., Schwartzman, S., Scott, P., Trow, M. (1994). La nueva producción del conocimiento. Barcelona, España: Pomares, corredor, S A.

Ghiso, Antonio. (1998). De la práctica singular al diálogo con lo plural: Aproximaciones a otros tránsitos y sentidos de la sistematización en épocas de globalización. Revista Latinoamericana de Educación, 1-9. Recuperado de: www.cepalforja.org/sistem/bvirtual/?p=661

Hare, R. M. (1999). Ordenando la ética: Una clasificación de las teorías éticas, Madrid, España: Editorial S.A.

Larrosa, J. (Ed). (2014). Experiencia y alteridad en educación. Rosario, Argentina: Homo Sapiens Ediciones.

Matus, T., Onetto, L., Rodríguez, N., Paiva, D., Ponce de León, M., Quesada, M. (1995). Perspectivas metodológicas en Trabajo Social. Recuperado de: www.ts.ucr.ac.cr/binarios/pela/pl-000380.pdf

Mejía, M. (2009). La sistematización como proceso investigativo o la búsqueda de la episteme de las prácticas. Revista Internacional Magisterio, № 33, 1-10 Recuperado de: http://www.cepalforja. org/sistem/sistem_old/sistematizacion_como_proceso_investigativo.pdf 
Netto, J. P. (Ed). (2003). Servicio social crítico, hacia la construcción de un nuevo proyecto ético político profesional, Sao Paulo, Brasil: Cortez.

Ranciere, J. (2003). El maestro ignorante. Barcelona, España: Editorial Laertes.

Schön, D. (1992). La formación de profesionales reflexivos: Hacia un nuevo diseño de la enseñanza y aprendizaje en las profesiones. Barcelona, España: Piados.

Sousa, J. (2008). La geopolítica del conocimiento y la gestión de procesos de innovación en la época histórica emergente. Documento de apoyo para el curso "Gestión de procesos, para la generación, apropiación, aplicación e implicaciones de conocimiento" de la Maestría en Conocimiento y Competitividad, Universidad Internacional del Ecuador. Recuperado de: http://www.apse.or.cr/ webapse/pedago/enint/souza08.pdf 